[0212-7199 (2004) 21: 4; pp 166-170] ANALES DE MEDICINA INTERNA Copyright (C) 2004 ARAN EDICIONES, S.L.

AN. Med INTERNA (Madrid) Vol. 21, N. ${ }^{\circ} 4$, pp. 166-170, 2004

\section{Patrones clínicos en neumonías comunitarias con documentación y sin documentación microbiológica. Estudio prospectivo y comparativo 1991-1997}

\author{
M. E. SÁNCHEZ GONZÁLEZ, J. GÓMEZ GÓMEZ1', J. GÓMEZ VARGAS, \\ L. MUÑOZ GIMENO, V. BAÑOS ${ }^{1}$, E. SIMARRO², J. RUIZ GÓMEZ ${ }^{2}$, M. CANTERAS $^{3}$, \\ M. VALDÉS ${ }^{4}$
}

Servicios de Urgencias, ${ }^{1}$ Medicina Interna-Infecciosas, ${ }^{2}$ Microbiología. Hospital Universitario Virgen de la Arrixaca. ${ }^{3}$ Departamento de Biestadística y ${ }^{4}$ Medicina Interna. Facultad de Medicina. Universidad de Murcia

\section{RESUMEN}

Introducción: La dificultad en el diagnóstico clínico de la NAC es uno de los problemas más importantes para su adecuado tratamiento.

Objetivos: Estudiar de forma prospectiva el patrón clínico de la NAC comparando las documentadas con las no documentadas microbiológicamente.

Pacientes y métodos: Hemos estudiado 409 pacientes diagnosticados de NAC ingresados en el HUVA entre enero de 1991 y mayo de 1997. Los criterios diagnósticos de neumonía y de ingreso hospitalario fueron los clásicos aceptados para estas infecciones. Se analizaron el patrón clínico de las NAC documentadas microbiológicamente de forma comparativa con las no documentadas y las documentadas se dividieron en típicas y atípicas de acuerdo con los microorganismos aislados. Se realizó estudio estadístico mediante tablas de contingencia y test exacto de Fisher.

Resultados: De todos ellos 409/161 (39,6\%) tenían documentación microbiológica, encontrándose 119 microorganismos típicos y 42 atípicos. El sexo mujer, la presencia de EPOC, fiebre, expectoración purulenta e hiperglucemia se asociaron significativamente con las NAC documentadas. La presencia de cardiopatía, situación clínica inicial mala, alteraciones gastrointestinales y existencia de infecciones y uso previo de antibióticos, se asociaron significativamente con las no documentadas.

La edad mayor de 65 años, presencia de comorbilidad, expectoración purulenta, dolor pleural junto con VSG $>50$ e infiltrado lobar lo encontramos asociado significativamente a las típicas, mientras que el hábito tabáquico y presencia de síntomas extrapulmonares (artromialgias) lo fueron con la atípicas.

Conclusiones: El conocimiento de los patrones clínicos de la NAC es de gran impacto en el diagnóstico precoz y tratamiento antibiótico adecuado.

PALABRAS CLAVE: Patrón clínico. Típico. Atípico. Neumonía comunitaria
THE CLINICAL PATRON OF THE NAC MICROBIOLOGICALLY DOCUMENTED COMPARE WITH THE NOT DOCUMENTED. PROS PECTIVE AND COMPARATIVE STUDY BETWEEN 1991 AND 1997

\section{ABSTRACT}

Introduction: The diagnostic of the pneumonia is the problems more important for its adequate treatment and best evolution.

Objectives: To study in a prospective way the patron clinic de la NAC compared the documented with the not documented in a microbiological way.

Patients y methods: We have studied patients with NAC hospitalized in the HUVA between January of 1991 and May of 1997. The diagnostical criterion of pneumonia and of the hospitable ingress were the classics accepted for this infections, was doing in all cases diagnostical studies not invasive.

It was analyzed the clinical patron of the NAC microbiologically documented compare to with the not documented. In the same way, the documented was divided in typical and not typical according to the microorganisms aisled. Besides was realized an statistic study using tables of contingency and test Fisher.

Results: It was studied 409 patients with NAC, from which 161 (39.6\%) had microbiological documentation, it was found 119 micro organism typical and 42 atypical. On the one hand, the female sex, EPOC, fever, tos and purulence esputum and hyperglucaemia, were associated significantly with the documented NAC. The presence of cardiopatía, seriously initial clinical situation, gastrointestinal disorders and previous infections and use of antibiotics, were associated with not documented.

The age more than 65 years, presence of comorbility, purulence sputum, pleural pain, toghether with VSG $>50$ and lobar infiltrate, were associated significantly to typical pneumonia, while tabáquical habit and extrapulmonary sintoms (artromialgias) were with the atypical.

Conclusions: The knowledge of the clinical patrons of the NAC are great impact in the diagnostic and treatment antibiotic adecuate.

KEY WORDS: Clinical patterns. Typical. Atypical. Community pneumonia.

Sánchez González ME, Gómez Gómez J, Gómez Vargas J, Muñoz Gimeno L, Baños V, Simarro E, Ruiz Gómez J, Canteras M, Valdés M. Patrones clínicos en neumonías comunitarias con documentación y sin documentación microbiológica. Estudio prospectivo y comparativo 1991-1997. An Med Interna (Madrid) 2004; 21: 166-170. 


\section{INTRODUCCIÓN}

De todas las infecciones adquiridas en la comunidad, la neumonía constituye un importante problema debido a los cambios que se están produciendo en la presentación clínica así como a la dificultad para alcanzar el diagnóstico microbiológico (1).

Para alcanzar el diagnóstico etiológico en la neumonía comunitaria es una pieza muy importante conocer la epidemiología del área geográfica en la que nos encontremos ya que en la mayor parte de los casos el tratamiento inicial va a ser empírico.

S. pneumoniae, siguen siendo en la actualidad la etiología más frecuente de las neumonías comunitarias $(2,3)$. Sin embargo, con mayor frecuencia aparecen otros microorganismos implicados en la etiología de la neumonía que se caracterizan por producir cuadros clínicos distintos a la presentación clásica y que aparecen frecuentemente en personas jóvenes y sin factores de riesgo asociados. Entre estos microorganismos destacan por su frecuencia Chlamydia pneumoniae que es el causante de entre el 6 y el 34\% de los casos de neumonía adquirida en la comunidad y Mycoplasma pneumoniae que en periodos epidémicos puede ser el origen de hasta un 50\% de los casos de neumonía. L. pneumophila, aparece en pacientes inmunocomprometidos y su frecuencia varía según áreas entre 5-17\% (4-6).

La descripción de los signos y síntomas clínicos llevó a hacer una clasificación clínico-etiológica de fácil manejo para el clínico pero que ha sido controvertida por los cambios que en la epidemiología de la neumonía se vienen produciendo en los últimos años debido fundamentalmente, al aumento en la edad de los pacientes y de las patologías crónicas que les afectan (7).

Ante las enormes dificultades del diagnóstico etiológico por la clínica y las posibles diferencias en la evolución entre las neumonías documentadas y no documentadas microbiologicamente, consideramos de interés la realización de un estudio para valorar la significación de los patrones clínicos en su diagnóstico y tratamiento empírico inicial, así como las posibles diferencias entre ambos grupos de neumonías.

\section{MATERIAL Y MÉTODO}

\section{SELECCIÓN DE LOS PACIENTES}

De los 1.121 pacientes mayores de 11 años ingresados en el Servicio de Medicina Interna del Hospital Universitario Virgen de la Arrixaca de Murcia en el periodo comprendido entre los meses de enero de 1991 y mayo de 1997, con el diagnóstico de neumonía adquirida en la comunidad hemos estudiado de forma prospectiva 409 de ellos.

Consideramos criterios de exclusión: la presencia de inmunodepresión grave debida a trasplantes, infección por virus de la inmunodeficiencia humana y fármacos inmunodepresores; la existencia de neoplasias; el ingreso inicial desde urgencias en unidades de cuidados intensivos y la hipersensibilidad conocida o supuesta a betalactámicos dado que el tratamiento empírico inicial de base estaba constituido por agentes betalactámicos.

\section{CRITERIOS DIAGNÓSTICOS}

Se consideró diagnóstico de neumonía la aparición de un infiltrado nuevo en la radiología de tórax y dos o más de los siguientes criterios: fiebre $\geq 37,8^{\circ} \mathrm{C}$, expectoración purulenta, leucocitosis $\geq 12.000 / \mathrm{mm}^{3} \mathrm{o} \leq 4.000 / \mathrm{mm}^{3}$, dolor torácico pleurítico y signos de condensación pulmonar a la exploración física.

Por último no debía existir antecedente de ingreso hospitalario dentro de las seis semanas previas al inicio del cuadro clínico.

Se consideraron como patógenos típicos, los microorganismos extracelulares más frecuentes en el tracto respiratorio (S. pneumoniae, H. Influenzae, M.catarrhalis y S. aureus) y como atípicos los intracelulares (M. pneumoniae, C. pneumoniae y L. pneumophila). Se definió como cuadro clínico tipo, el que presentaba un comienzo brusco de menos de 48 horas de evolución, fiebre superior a $37,8^{\circ} \mathrm{C}$ y presencia de tos productiva, expectoración purulenta y dolor torácico. Atípico, fiebre superior a $37,8{ }^{\circ} \mathrm{C}$, tos irritativa y manifestaciones extrapulmonares (artromialgias, vómitos, diarreas, alteración estado conciencia, cefalea).

\section{ESTUDIO MICROBIOLÓGICO}

Para el diagnóstico microbiológico se usaron exclusivamente procedimientos no invasivos. Así en todos los pacientes se realizaron tomas de suero para hemocultivos seriados durante el pico febril. Si existía expectoración se realizaba toma de muestra para tinción de gram y cultivo de la misma considerándose válidas cuando mostraban más de 25 leucocitos y menos de 8 células escamosas por campo. Se tomaron también muestras de suero para la realización de técnicas serológicas con el fin de identificar anticuerpos frente a Mycoplasma pneumoniae, Chlamydia pneumoniae, Legionella pneumophila y Coxiella burnetti. Asimismo se realizó estudio de antígeno urinario para Legionella.

Si existía derrame pleural se procedía al drenaje y cultivo del mismo.

\section{DIAGNÓSTICO MICROBIOLÓGICO}

Consideramos que el diagnóstico microbiológico era probable o confirmado cuando el microorganismo se aislaba en el suero o en el líquido pleural, cuando se aislaba en el cultivo de esputo con una tinción de gram compatible o si existía un aumento del cuádruplo del título de anticuerpos frente a $C$. pneumoniae, $M$. pneumoniae y L. pneumophilla, o positividad de los antígenos urinarios para Legionella.

\section{EPIDEMIOLOGÍA Y CLÍNICA}

En el protocolo de recogida de datos se incluían entre otros los referentes a edad, sexo, infecciones y consumo de antibióticos previos al ingreso, enfermedades crónicas concomitantes, hábitos tóxicos, considerando como hábito tabaquico el consumo de más de 10 paquetes de cigarrillos al año y alcohólico el consumo de $100 \mathrm{~g} / \mathrm{d}$ en hombre y $80 \mathrm{~g} / \mathrm{d}$ en mujeres, inicio del cuadro clínico, existencia de datos clínicos cómo tos, fiebre, expectoración, dolor torácico, artromialgias, alteraciones del hábito gastrointestinal, del estado de conciencia, así como la presencia de complicaciones durante la estancia en el hospital. 
Definimos insuficiencia respiratoria como una $\mathrm{PaO}_{2}$ inferior a $60 \mathrm{~mm} \mathrm{Hg}$ y/o una $\mathrm{PCO}_{2}$ superior a $50 \mathrm{mmHg}$. Definimos fracaso renal cuando existía un aumento en la concentración de creatinina superior a $2 \mathrm{mg} / \mathrm{dl}$ en sujetos con función renal normal y shock séptico como una cifra de presión arterial sistólica inferior a $90 \mathrm{mmHg}$ y oliguria.

Clasificamos el estado clínico inicial atendiendo a los criterios de Winston (8): crítico, cuando existe alteración grave hemodinámica y probabilidad de muerte en las primeras 24horas; grave, cuando la probabilidad de muerte puede producirse en los días posteriores; regular, cuando no existe probabilidad de muerte; estable, sin afectación general.

\section{EVOLUCIÓN}

A todos los enfermos se le realizaban exámenes clínicos diarios durante el ingreso y también exploraciones analíticas y microbiológicas. Clasificamos la evolución cómo curación, recurrencia, fracaso en el tratamiento y muerte según los criterios clásicamente admitidos en la literatura.

\section{ANÁLISIS ESTADÍSTICO}

Los datos obtenidos fueron analizados mediante análisis bivariante, con tablas de contingencia, coeficiente de correlación de Pearson y test exacto de Fisher. Se consideraron como variables dependientes el tipo de patrón clínico (típico y atípico), grupo de neumonías(documentadas y no documentadas) y el tipo de evolución (curación, exitus) entre ambos grupos de neumonías.

\section{RESULTADOS}

\section{DESCRIPCIÓN GENERAL Y MICROBIOLOGÍA}

De todos los pacientes estudiados (409) un 59,66\% de ellos eran varones y el $40,34 \%$ mujeres con una edad media de 61,7 años (DE+ 17,48). De ellos 213 (52,08\%), habían presentado infección respiratoria en las cuatro semanas anteriores al ingreso hospitalario, existiendo en 205 pacientes $(50.12 \%)$ uso previo de algún tipo de antibiótico en ese mismo periodo de tiempo.

Las enfermedades de base más frecuentemente encontradas fueron: la enfermedad pulmonar crónica $(30,56 \%)$, la cardiopatía $(22,00 \%)$, la hipertensión arterial $(22,74 \%)$ y la diabetes mellitus $(17,36 \%)$. La menos frecuentes incluían: hepatopatía $(6,36 \%)$ y nefropatía $(2,44 \%)$.

Con relación a los hábitos tóxicos de nuestros pacientes vimos que un $54,76 \%$ de los mismos eran fumadores activos $\mathrm{y}$ un $16,62 \%$ consumidores habituales de alcohol (superior a $100 \mathrm{~g} / \mathrm{d}$ en varones y $80 \mathrm{~g} / \mathrm{d}$ en mujeres).

La evolución fue favorable en la mayoría de los pacientes, alcanzando la curación el 90,22\%,

\section{CARACTERÍSTICAS MICROBIOLÓGICAS}

De todos los pacientes incluidos en nuestro estudio, llegamos al aislamiento del microorganismo causante de la neumo- nía en 161 casos, lo que supuso un 39,36\% de los estudiados. De ellos 119 correspondían a microorganismos considerados como típicos y 42 como atípicos. Las técnicas microbiológicas donde se obtuvieron fueron las siguientes: hemocultivos (29), cultivo de liquido pleural (12), esputo (78), antígeno urinario de Legionella (7) y serología (35). Los microorganismos se distribuyeron como: 85 Streptococcus pneumoniae; 30 Haemophilus influenzae; 24 Chlamydia pneumoniae; 11 Mycoplasma pneumoniae; 7 Legionella pneumophila; 3 Staphilococcus aureus; 1 Moraxella catarrhalis. No se detectaron neumonías de etiología múltiple, causadas por varios microorganismos.

Streptococcus pneumoniae, presentaba en el 50,59\% de los casos una resistencia intermedia a la penicilina (CIM 0,1-1 $\mathrm{mg} / \mathrm{ml})$.

\section{ESTUDIO COMPARATIVO ENTRE PACIENTES CON O SIN DOCUMENTACIÓN MICROBIOLÓGICA}

Entre las diferencias más significativas entre ambos grupos de pacientes con neumonías comunitarias encontramos al sexo varón $(\mathrm{p}<0,025)$, enfermedad pulmonar obstructiva crónica $(\mathrm{p}<0,05)$, presentación clínica con tos $(\mathrm{p}<0,025)$, fiebre $(\mathrm{p}<0,001$; expectoración $(\mathrm{p}<0,01)$, situación clínica inicial regular $(\mathrm{p}<0,05)$, cifras elevadas de glucemia $(\mathrm{p}<0,05)$ y mayor necesidad de ventilación mecánica $(\mathrm{p}<0,001)$, asociados con una documentación microbiológica positiva. No se encontraron diferencias significativas entre los hábitos tóxicos.

Los antecedentes de infección respiratoria y uso previo de antibióticos en las cuatro semanas anteriores $(p<0,001)$, presencia de enfermedades cardiacas $(\mathrm{p}<0,05)$, situación clínica inicial mala $(\mathrm{p}<0,05)$, junto con la existencia de alteraciones en el estado mental a la exloración física $(\mathrm{p}<0,01)$, alteraciones del hábito gastrointestinal $(\mathrm{p}<0,001 ;)$ y la alteración en las pruebas de función hepática $(\mathrm{p}<0,001)$ se relacionaban de forma estadísticamente significativa con la ausencia de documentación microbiológica $(\mathrm{p}<0,001)$.

No encontramos diferencias significativas entre los patrones radiológicos de ambos grupos de neumonías, así como en su mortalidad ( 10 y 14, respectivamente) (Tabla I).

\section{ESTUDIO COMPARATIVO ENTRE PACIENTES CON NEUMONÍAS CAUSADAS POR MICROORGANISMOS TÍPICOS Y ATÍPICOS}

La edad superior a 65 años $(\mathrm{p}<0,001)$, enfermedades de base crónica, diabetes $(p<0,01)$, enfermedad pulmonar obstructiva crónica $(\mathrm{p}<0,05)$, cardiopatía $(\mathrm{p}<0,05)$, existencia de expectoración $(\mathrm{p}<0,05)$, dolor torácico de características pleuríticas $(\mathrm{p}<0,05)$, patrón radiológico de afectación lobar $(\mathrm{p}<0,05 ;)$ y una velocidad de sedimentación globular superior a $50 \mathrm{~mm}$ en la primera hora $(\mathrm{p}<0,005)$ se encontraron asociados significativamente con microorganismos típicos.

Por el contrario, el hábito tabáquico $(\mathrm{p}<0,05)$ y presencia de artromialgias $(\mathrm{p}<0,05)$ en la presentación clínica se encontró asociado significativamente con los microorganismos atípicos. No encontramos diferencias significativas de gravedad clínica inicial y de mortalidad (6 / 4) entre las neumonías típicas y atípicas (Tabla II). 
TABLA I

DIFERENCIAS ESTADÍSTICAMENTE SIGNIFICATIVAS ENTRE LAS CARACTERÍSTICAS GENERALES DE LOS GRUPOS DE NEUMONÍAS

\begin{tabular}{|c|c|c|c|}
\hline & Documentadas & No documentadas & $p$ \\
\hline \multicolumn{4}{|l|}{ Sexo: } \\
\hline Varón & 107 & 137 & \\
\hline Mujer & 54 & 111 & $<0,025^{*}$ \\
\hline \multicolumn{4}{|c|}{ Enfermedad de base: } \\
\hline EPOC & 60 & 65 & $<0,05^{*}$ \\
\hline Cardiopatía & 26 & 64 & $<0,05^{* *}$ \\
\hline Inf.previas: & 55 & 138 & $<0,001^{* *}$ \\
\hline Ant. previos & 56 & 133 & $<0,001^{* *}$ \\
\hline \multicolumn{4}{|l|}{ Clínica } \\
\hline Tos & 145 & 201 & $<0,025^{*}$ \\
\hline Fiebre & 161 & 218 & $<0,001^{*}$ \\
\hline Expectoración & 104 & 123 & $<0,01^{*}$ \\
\hline Alt.g-i & 10 & 44 & $<0,001^{* *}$ \\
\hline \multicolumn{4}{|c|}{ Situación clínica inicial } \\
\hline Mala & 45 & 92 & $<0,05^{* *}$ \\
\hline Regular & 112 & 146 & $<0,05^{*}$ \\
\hline Complicaciones & & & \\
\hline $\begin{array}{l}\text { V. mecánica } \\
\text { Analítica }\end{array}$ & 8 & 2 & $<0,01^{*}$ \\
\hline \multirow{2}{*}{\multicolumn{4}{|c|}{ Actividad de protrombina }} \\
\hline & & & \\
\hline Normal & 156 & 222 & \\
\hline $\begin{array}{l}\text { Alterada } \\
\text { Glucemia }\end{array}$ & 6 & 25 & $<0,02^{*}$ \\
\hline \multicolumn{4}{|l|}{ Glucemia } \\
\hline$>120$ & 60 & 68 & \\
\hline$<120$ & 102 & 179 & $<0,05^{*}$ \\
\hline \multicolumn{4}{|l|}{ Enzimas hepáticos } \\
\hline Alterados & 30 & 83 & \\
\hline Normales & 132 & 164 & $<0,001^{* *}$ \\
\hline
\end{tabular}

Enf. base: enfermedad de base; EPOC: enfermedad pulmonar obstructiva crónica; DM: diabetes mellitus; HTA: hipertensión arterial; Inf.previas:infecciones previas; Antib.previos: Uso previo de antibióticos en las seis semanas anteriores; Alt. g-i: alteraciones gastrointestinales; V. mecánica: ventilación asistida;

P: nivel de significación; NS: no significativo; * : existen diferencias estadísticamente significativas a favor de la documentación microbiológica; **: existen diferencias estadísticamente significativas a favor de la no documentación microbiológica.

\section{DISCUSIÓN}

Las neumonías adquiridas en la comunidad que precisan ingreso hospitalario se producen fundamentalmente en personas mayores de 60 años con patologías crónicas debilitantes y en consumidores habituales de tabaco y alcohol, lo que coincide con lo ya publicado por otros autores $(9,10)$.

La etiología de las neumonías adquiridas en la comunidad no es bien conocida y entre un 30 y un $60 \%$ de los casos no se llega a alcanzar el diagnóstico etiológico (11). En nuestro estudio se alcanzó un porcentaje cercano al $40 \%$ de documentación microbiológica que lo podemos considerar aceptable
TABLA II

DIFERENCIAS SIGNIFICATIVAS DE LOS PACIENTES CON NEUMONÍAS DOCUMENTADAS

\begin{tabular}{lccc}
\hline & Típicas & Atípicas & $p$ \\
\hline Edad: & & & \\
$>65 a$ & 87 & 14 & \\
$<65$ a & 32 & 28 & $<0,001^{*}$
\end{tabular}

\section{Enf. base:}

EPOC

Cardiopatía

DM

$\begin{array}{lcl}50 & 10 & <0,05^{*} \\ 25 & 1 & <0,05^{*} \\ 27 & 2 & <0,01^{*}\end{array}$

Hábitos tóxicos:

Tabaco $61 \quad 28<0,05^{* *}$

Clínica:

$\begin{array}{lccc}\text { Expectoración } & 80 & 24 & <0,05^{*} \\ \text { Dolor pleurítico } & 56 & 12 & <0,05^{*} \\ \text { Artromialgias } & 10 & 8 & <0,05^{*}\end{array}$

Analítica VSG:

$\begin{array}{llll}>50 & 87 & 19 & <0,005^{*} \\ <50 & 33 & 23 & \end{array}$

Tipo infiltrado:
Lobar
38
7
$<0,05^{*}$

*: Existen diferencias estadísticamente significativas a favor de microorganismos típicos; **: Existen diferencias estadísticamente significativas a favor de microorganismos atípicos

teniendo en consideración que no se utilizaron técnicas invasivas para su diagnóstico en los sujetos y que un elevado número de pacientes había consumido algún tipo de tratamiento antibiótico antes del ingreso en nuestro centro, lo que reduce de forma significativa la posibilidad de aislamiento del microorganismo.

Haciendo referencia a los resultados que obtuvimos y en los que se relacionaba la existencia de alteraciones en el hábito gastrointestinal y la elevación de las enzimas hepáticas con la no documentación microbiológica, no encontramos ninguna referencia en la bibliografía publicada y quizá pudieran explicarse con la asociación de éstos parámetros con una etiología atípica de la neumonía y su dificultad para el aislamiento de estos tipos de microorganismos.

Parece claro que la mayor facilidad para expectorar de los varones respecto a las mujeres y la concomitancia de enfermedad crónica pulmonar, explica la relación de estos tipos de pacientes y la existencia de aislamiento del agente etiológico de la neumonía comunitaria.

En relación con los patrones clínicos, nuestros resultados que muestran una asociación significativa entre la presencia de expectoración purulenta y dolor torácico de características pleuríticas junto con un patrón radiológico de condensación lobar, con una etiología por microorganismos típicos de neumonía comunitaria, son diferentes a lo publicado por otros autores (12). Estas diferencias pueden estar en relación al incluir en estos estudios diferentes grupos de enfermos, así 
como los casos de adquisición nosocomial en relación con las neumonía por S. pneumoniae y por L. pneumophila, al contrario que en nuestro trabajo que solo analizamos las adquiridas en la comunidad y además se excluyeron los pacientes inmunodeprimidos graves, así como los casos con ingreso inicial en UCI.

La aparición de artromialgias en la clínica de las neumonías, al igual que otros estudios (13-16) la encontramos asociada significativamente con los microorganismos atípicos.

En cuanto a las exploraciones complementarias realizadas hemos de destacar que existía asociación estadística entre un aumento de los reactantes de fase aguda como la velocidad de sedimentación globular y las neumonías producidas por microorganismos típicos, al igual que fue observado en otros estudios (7).

Respecto al desarrollo de complicaciones como insuficiencia respiratoria, insuficiencia renal, y shock séptico, no existían en nuestro estudio, diferencias entre las documentadas y no documentadas microbiológicamente, ni entre neumo- nías causadas por microorganismos típicos o atípicos. Por el contrario, la mayor necesidad de ventilación mecánica la encontramos asociada significativamente con los pacientes con neumonía documentada microbiologicamente.

Ante estos resultados, consideramos que el patrón clínico de las neumonías comunitarias es de gran importancia en su diagnóstico inicial y elección del tratamiento antibiótico más adecuado y racional.

En los casos de patrón clínico dudoso, o situación clínica inicial muy grave, el mayor conocimiento de la epidemiología de cada área nos puede ayudar a su selección, sin embargo la gravedad de diversos patógenos, especialmente L. pneumophila, S. pneumoniae resistente a penicilina y en menor frecuencia gramnegativos, obligan al clínico a realizar de forma empírica un tratamiento antibiótico de combinación entre betalactámico + macrólido o monoterapia con levofloxacino a dosis elevadas, en espera de los resultados microbiológicos, ya que el tratamiento inadecuado se asocia con peor evolución $(17,18)$.

\section{Bibliografía}

1. Falguera M, Gudiol F, Sabria M, Álvarez- Lerma F, Cordero E. Infecciones tracto respiratorio inferior. Neumonía adquirida en la comunidad. En JM Aguado, B. Almirante y J. Fortum Eds.) Protocolos Clínicos SEIMC. SmithKline-Beecham Madrid 2000: Vol-1: 11-17.

2. Bartlett J, Mundy L. Community-acquired pneumonia. N Engl J Med 1995; 333 (24): 1618-1623.

3. Alfageme I, Muñoz J, de la Cruz I. Neumonía comunitaria. Epidemiología. Factores de riesgo y pronóstico. Arch Bronconeumol 1998; 34 (Supl. 2): 17-24.

4. Almirante J, Bolíbar I. Aspectos epidemiológicos no resueltos de la neumonía adquirida en la comunidad en adultos. Med Clin (Barc) 1998; 111: 216-217.

5. Almirante B, Bolívar I, Vidal J, Sauca G, Coll P, Niklasson B, et al Epidemiology of community-acquired pneumonia in adults: a populationbased study. Eur Respir J 2000; 15: 757-763.

6. Sopena N, Sabria M, Pedro-Botet ML, Manterola JM, Matas L, Dominguez J, et al. Prospective study of community-acquired pneumonia of bacterial origin in adults. Eur J Clin Microbiol Infect Dis 1999; 18: 852858.

7. Molinos L, Fernández R, Gullón JA, Rubinos G, Alonso MA, Escudero $\mathrm{C}$, et al. Neumonía adquirida en la comunidad con tratamiento hospitalario. Interés de la clínica y exámenes complementarios en la predicción de la etiología. Arch Bronconeumol 1997; 33: 230-234.

8. Winston DJ, Murphy W, Young LS, Hewitt WL. Piperacillin Therapy for serious bacterial infections. Am J Med 1980; 69: 255-261.

9. Fine M, Auble T, Yealy D, Hanusa BH, Weissfeld LA, Singer DE, et al A prediction rule to identify low risk patients with community-acquired pneumonia. N J Engl Med 1997; 336: 243-250.

10. File T, Tan J Incidence, etiologic pathogens, and diagnostic testing of community-acquired pneumonia. Curr Opin Pulm Med 1997; 3: 8997.

11. Gómez J, Baños V, Ruiz J, Soto MC, Muñoz L, Núñez ML, et al. Prospective study of epidemiology and prognostic factors in communityacquired pneumonia. Eur J Clin Microbiol Infect Dis 1996; 15: 556560 .

12. Granados A, Podzamczer D, Gudiol F, Manresa F. Pneumonia due to Legionella pneumophila and pneumococcal pneumonia: simmilarities and differences on presentation. Eur Respir J 1989; 2: 130-134.

13. Irigaray R, Dorca J. Neumonías atípicas. Arch Bronconeumol 1996; 32 1887-195.

14. Lieberman D, Ben-Yaakov M, Lazarovich Z, Porath A, Sachlaeffer F, Leinonen M, et al. Chlamydia pneumoniae community-acquired pneumonia: a review of 62 hospitalized adult patients. Infection 1996; 24 : 109-114.

15. Lieberman D, Schlaeffer F, Horowitz S, Horovitz O, Porath A. Mycoplasma pneumoniae community-acquired pneumonia: a review of 101 hospitalized adult patients. Respiration 1996; 63 (5): 261-266.

16. Lieberman D, Schlaeffer F, Boldur I, Horowitz S, Friedamn MG, Leinonen M, et al. Multiple pathogens in adult patients amitted with community-acquired pneumonia: a one year prospective study of 346 consecutive patients. Thorax 1996; 51: 179-184.

17. Metlay JP. Update on community-acquired pneumonia: impact of antibiotic resistanse on clinical outcomes. Curr Opin Infect Dis 2002; 15: 163-7.

18. Metersky ML. Community-acquired pneumonia: process of care studies. Current Opin Infect Dis 2002; 15: 169-174. 